\title{
Mutual comparative analysis: a new topography-guided custom ablation protocol referencing subjective refraction to modify corneal topographic data
}

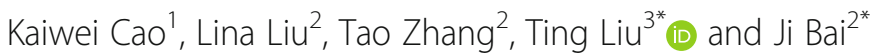

\begin{abstract}
Background: Several planning algorithms have been developed for topography-guided custom ablation treatment (T-CAT), but each has its own deficiencies. The purpose of this study is to demonstrate the potential of a novel mutual comparative analysis (MCA) informed by manifest refraction and corneal topographic data and the patient's subjective perception in correcting ametropia.

Methods: This retrospective review included patients with significant preoperative differences in the power or axis of astigmatism according to the manifest refraction and corneal topographic data (power $>0.75 \mathrm{D}$ and/or axis > $10^{\circ}$ ). T-CAT planning was designed using MCA. Follow-ups were conducted for at least 6 months.

Results: Seventy-nine patients (121 eyes) were included. The mean preoperative deviation in the astigmatic power and axis were $0.72 \pm 0.43 \mathrm{D}$ and $20.18 \pm 23.68^{\circ}$, respectively. The average oculus residual astigmatism (ORA) was $0.81 \pm 0.32 \mathrm{D}$ (range: $0.08-1.66 \mathrm{D}$ ). Six months postoperatively, the mean spherical equivalent refraction was $0.04 \pm$ $0.42 \mathrm{D}$, and the mean cylinder was $-0.27 \pm 0.24 \mathrm{D}$. The mean efficacy and safety indices were 1.10 and 1.15 , respectively. The uncorrected distance visual acuity in $92 \%$ of the eyes was the same or better than the corrected distance visual acuity. The angle of error was $\pm 5^{\circ}$ in $61 \%$ of eyes and $\pm 15^{\circ}$ in $84 \%$ of eyes. Residual astigmatism was $\leq 0.5 \mathrm{D}$ in $91 \%$ of eyes. Optical quality and photopic contrast sensitivity did not change significantly $(p>0.05)$, and the scotopic contrast sensitivity decreased at 3,6 , and $12 \mathrm{cpd}(p<0.05)$. The vertical coma and horizontal coma of the anterior corneal surface significantly decreased postoperatively but increased during follow-up.

Conclusions: The MCA demonstrated safety, efficacy, accuracy, predictability, and stability and can be used as a complementary and feasible method for T-CAT.
\end{abstract}

Keywords: Mutual comparative analysis, Topography-guided custom ablation treatment, Astigmatism, Manifest refraction, Topography

\footnotetext{
*Correspondence: tingliu1597@126.com; baijidaping@163.com

${ }^{3}$ Department of Ophthalmology, Daping Hospital and the Research Institute

of Surgery of the Army Medical University of PLA, No. 10 Changjiangzhi

Road, Yuzhong District, Chongqing 400042, China

${ }^{2}$ Chongqing vision institute, No. 77 Changjiang 2 Road, Yuzhong District,

Chongqing 400042, China

Full list of author information is available at the end of the article
}

(c) The Author(s). 2020 Open Access This article is licensed under a Creative Commons Attribution 4.0 International License, which permits use, sharing, adaptation, distribution and reproduction in any medium or format, as long as you give appropriate credit to the original author(s) and the source, provide a link to the Creative Commons licence, and indicate if changes were made. The images or other third party material in this article are included in the article's Creative Commons licence, unless indicated otherwise in a credit line to the material. If material is not included in the article's Creative Commons licence and your intended use is not permitted by statutory regulation or exceeds the permitted use, you will need to obtain permission directly from the copyright holder. To view a copy of this licence, visit http://creativecommons.org/licenses/by/4.0/ The Creative Commons Public Domain Dedication waiver (http://creativecommons.org/publicdomain/zero/1.0/) applies to the data made available in this article, unless otherwise stated in a credit line to the data. 


\section{Background}

Developed over the course of two decades, topographyguided custom ablation treatment (T-CAT) $[1,2]$ was initially used for the retreatment of irregular corneas after corneal refractive surgery $[3,4]$ and the treatment of keratectasia with collagen cross-linking [5].

In 2013, the U.S. Food and Drug Administration (FDA) approved the Contoura vision (CV) method, the WaveLight Excimer Laser System with WaveLight Topolyzer, and a treatment planning software for topographyguided laser-assisted in situ keratomileusis (LASIK) treatment [6].

Several planning algorithms have been developed for the CV system. The FDA algorithm uses manifest refraction as the correction amount during the operation, while a modified FDA algorithm determines the astigmatic axis of the correction based on corneal topographic data. However, the FDA algorithm needs to satisfy the following conditions: (1) for patients with astigmatisms of $>2.00 \mathrm{D}$, the difference in the astigmatic axis between manifest refraction and topographic data should not exceed 5 degrees, (2) for patients with astigmatisms of $<1.75 \mathrm{D}$, the difference in the astigmatic axis between the manifest refraction and topographic data should not exceed 10 degrees, and (3) the difference in astigmatic power between manifest refraction and topographic data should not exceed $0.75 \mathrm{D}$. Due to corneal irregularity, asymmetric astigmatism, or astigmatism in the eye, the power and axis of the astigmatism are sometimes different between manifest refraction and topographic data. The topographymodified refraction (TMR) [7] and Layer Yolked Reduction of Astigmatism (LYRA) protocols [8] have been reported using astigmatic data measured by corneal topography as the correction amount of astigmatism. The LYRA protocol is applied when the manifest refraction and topographic data differ largely in terms of the power and axis of the astigmatism.

Multiple factors can result in a difference in the manifest refraction and topographic astigmatism. Astigmatism correction based on corneal topography alone has deficiencies when the posterior corneal surface or intraocular astigmatism is large [9]. Proposed by Alpins [10], vector planning allows for the combination of manifest refraction and corneal topographic data to correct astigmatism.

Informed by previous methods, we devised a new topography-guided custom ablation protocol to correct refractive errors. Mutual comparative analysis (MCA) combines manifest refraction and corneal topographic data and the patient's subjective perception. Here, we demonstrated the potential of MCA to correct ametropia.

\section{Methods}

This retrospective study reviewed 121 eyes of 79 patients (29 men) in which myopia and astigmatism were corrected with T-CAT LASIK or T-CAT laser-assisted subepithelial keratectomy (LASEK) at Daping Hospital, Army Medical University of PLA, between August 2016 and August 2018. The preoperative power and/or axis of the astigmatism in these eyes differed between the manifest refraction and corneal topographic data (power $>$ $0.75 \mathrm{D}$ and/or axis $>10$ degrees). Follow-up was conducted in each case for at least 6 months. Visual acuity and refraction were recorded, and the objective scattering index (OSI), cutoff for modulation transfer function (MTF), and Strehl ratio (SR) were measured with Optical Quality Analysis System II (OQAS, Visiometrics, Cerdanyola del Vallès, Spain). Contrast sensitivity testing was accomplished using the CSV-1000E (VectorVision, USA). The Pentacam (OCULUS, Germany) is a threedimensional image scanner and can be used for comparing images at different time points, whereas Topolyzer is a two-dimensional image scanner covering only the anterior surface of the cornea and cannot be used for comparing images at different time points. Since Topolyzer is infrequently used in postoperative examinations, corneal high order aberrations (HOAs) were measured with Pentacam.

The corneas of all patients were preoperatively scanned and processed with Topolyzer Vario and Contoura WaveNet, respectively. Procedures were performed with the Wavelight EX500 Laser (Alcon, US). All surgeries were conducted by the same surgeon (Ji Bai). Surgical planning was completed in the $\mathrm{CV}$ system according to the MCA algorithms.

Patients were instructed to wear the bandage soft contact lens for 1 week after receiving LASEK. Antiinfection (levofloxacin eye drops, Santen, Japan; three times daily for 3-4 weeks) and anti-inflammatory (0.5\% loteprednol etabonate ophthalmic suspension, Bausch \& Lomb, USA; four times daily for 1 week, followed by twice daily for 3 to 4 weeks) treatments were prescribed to each patient in addition to the routine postoperative administration of sodium hyaluronate eye drops (URSAPHARM Arzneimittel GmbH, Germany; four times daily from the first week post-surgery to the third month post-surgery).

All statistical analyses were performed using SPSS 25.0 (IBM, US). Optical quality, contrast sensitivity, and HOAs were compared using paired t-tests. Visual acuity and refraction analyses were performed according to the Standard Graphs for Reporting Refractive Surgery [11]. Standard graphs for reporting outcomes for correcting astigmatism were based on the Alpins Method, and the single angle polar plots were analyzed by AstigMATIC [12].

\section{The MCA}

Corrected visual acuity is a psychophysical response of visual perception and cognition. Through our clinical 
observations, due to a variety of factors (such as regulation of the ciliary muscle), best-corrected distance visual acuity (BCDVA) is achieved in a refractive range rather than as a fixed value. For example, while astigmatism between -1.00 diopter cylinder (DC) to $-1.50 \mathrm{DC}$ does not affect patient visual acuity, visual acuity decreases significantly or subjective perception deteriorates if a patient's astigmatism is beyond the aforementioned range. The astigmatism axis is subject to a similar phenomenon. Although axis adjustment within a certain range has little effect on the visual perception of patients, patients cannot endure axis adjustments beyond the range. Due to regulation, cognitive ability, previous corrective glasses and other factors, the range varies from patient to patient. This feature is the basis of MCA. Based on TMR data (topographic astigmatic power and axis), we set the TMR data as the target and mutually compared TMR data with the manifest refraction.

The MCA is conducted as follows:

1) Optometry and corneal topography examination

2) A difference in the power or axis of astigmatism between manifest refraction and topographic data that exceeds the application conditions of the FDA algorithm

3) TMR data used as the "target," which can be converted into a frame glasses diopter. Using a phoropter, start with $1 / 2$ or $1 / 3$ TMR or other values, adjust the manifest refractive power and axis of astigmatism so that they approach the "target" while paying attention to the change of the spherical equivalent (SE) and observe the patient's subjective perception and corrected visual acuity

4) When the power or axis position exceeds a specific value, which varies from patient to patient, corrected visual acuity decreases significantly, or subjective perception deteriorates. This value is used as the correction amount of the surgery

5) If the "target" value (TMR data) is reached and the patient still has no obvious discomfort, then the TMR data are used as the correction amount.

\section{Results}

Of the 121 eyes, 22 and 99 eyes underwent T-CAT LASEK and T-CAT LASIK, respectively. Demographic characteristics are shown in Table 1. Slit-lamp examinations in all patients were normal.

The average deviation of astigmatic power from the manifest to topographic data was $0.72 \pm 0.43 \mathrm{D}$ (range: $0.03-1.93 \mathrm{D})$. The average deviation of the axis was $20.18 \pm 23.68$ degrees (range: 0 to 89 degrees). The average oculus residual astigmatism (ORA) was $0.81 \pm$ $0.32 \mathrm{D}$ (range: 0.08 to $1.66 \mathrm{D}$ ). All astigmatism values
Table 1 Demographic characteristics

\begin{tabular}{lllll}
\hline & Patients & Sex (Male/Female) & Eyes & Age (years) \\
\hline LASIK & 65 & $23 / 42$ & 99 & $27.32 \pm 6.83$ \\
LASEK & 14 & $6 / 8$ & 22 & $24.77 \pm 7.20$
\end{tabular}

$L A S I K=$ laser-assisted in situ keratomileusis, $L A S E K=$ laser-assisted subepithelial keratectomy

were expressed as negative values. Preoperative data on refractive and visual acuity and correction data are shown in Table 2. Among them, 18 eyes (14.88\%) tolerated $100 \%$ TMR, 30 eyes (24.79\%) tolerated $75-100 \%$ TMR, 48 eyes (39.67\%) tolerated 50-75\% TMR, 16 eyes (13.22\%) tolerated $25-50 \%$ TMR, and 9 eyes (7.44\%) tolerated $0-25 \%$ TMR.

\section{General conditions}

General conditions such as keratometry, central corneal thickness (CCT), and intraocular pressure (IOP) preoperatively and 6 months postoperatively are summarized in Table 3.

\section{Visual acuity and refraction}

From all 121 eyes, the uncorrected distance visual acuity (UDVA) was 1.0 or better in $96(79 \%)$ eyes and 0.80 or better in 120 (99\%) eyes (Fig. 1a). The mean UDVA was $1.07 \pm 0.22$ (range, 0.6-2.0). The UDVA was within one line of the CDVA in all eyes (Fig. 1b). The mean efficacy index (postoperative UDVA/preoperative CDVA) of all treatments was $1.10 \pm 0.16$ (range, 0.75-1.67) after 6 months. The mean postoperative and preoperative CDVAs of all eyes were $1.12 \pm 0.21$ (range, 0.6-2.0) and $0.98 \pm 0.18$ (range, 0.6-1.5), respectively. The mean safety index (postoperative CDVA/preoperative CDVA) was $1.15 \pm 0.17$ (range, 0.75-1.67) in all eyes. One eye (1\%) lost one line of CDVA (Fig. 1c). This case underwent T-CAT LASEK and exhibited corneal haze. There was a high correlation between the attempted and

Table 2 Preoperative data on refractive and visual acuity and corrected data

\begin{tabular}{lll}
\hline & Mean \pm SD & Range \\
\hline Sphere $(M)(D)$ & $-5.29 \pm 1.64$ & -0.75 to -9.00 \\
Cylinder $(M)(D)$ & $-0.78 \pm 0.68$ & -5.00 to 0.00 \\
Axis $(M)\left(^{\circ}\right)$ & $81.74 \pm 69.46$ & 0.00 to 180 \\
CDVA & $0.88 \pm 0.13$ & 0.10 to 1.0 \\
UDVA & $0.16 \pm 1.89$ & 0.02 to 1.0 \\
Cylinder (T)(D) & $-1.43 \pm 0.99$ & -5.89 to -0.02 \\
Axis (T) $\left(^{\circ}\right)$ & $75.77 \pm 79.52$ & 0.00 to 180 \\
Sphere $(C)(D)$ & $-5.14 \pm 1.59$ & -9.00 to -0.75 \\
Cylinder (C)(D) & $-0.99 \pm 0.80$ & -5.25 to 0.00 \\
Axis (C) $\left(^{\circ}\right)$ & $75.20 \pm 72.39$ & 0.00 to 180.00 \\
\hline
\end{tabular}

$M=$ manifest, $T=$ topographic, $C=$ corrected, $C D V A=$ corrected distance visual acuity, UDVA= uncorrected distance visual acuity 
Table 3 General condition of preoperatively and postoperative 6 months

\begin{tabular}{lllll}
\hline & K1 $(\mathrm{D})$ & $\mathrm{K} 2(\mathrm{D})$ & $\mathrm{CCT}(\mu \mathrm{m})$ & $\mathrm{IOP}(\mathrm{mmHg})$ \\
\hline Preoperatively & $43.05 \pm 1.60$ & $44.3 \pm 1.58$ & $540.61 \pm 25.24$ & $15.21 \pm 2.67$ \\
Postoperative 6 months & $38.76 \pm 2.09$ & $39.43 \pm 2.17$ & $464.06 \pm 30.9$ & $10.71 \pm 1.75$ \\
$p$ & 0.000 & 0.000 & 0.000 & 0.003 \\
\hline
\end{tabular}

$K 1=$ keratometry flat, $K 2=$ keratometry steep, $C C T=$ central corneal thickness, $I O P=$ intraocular pressure

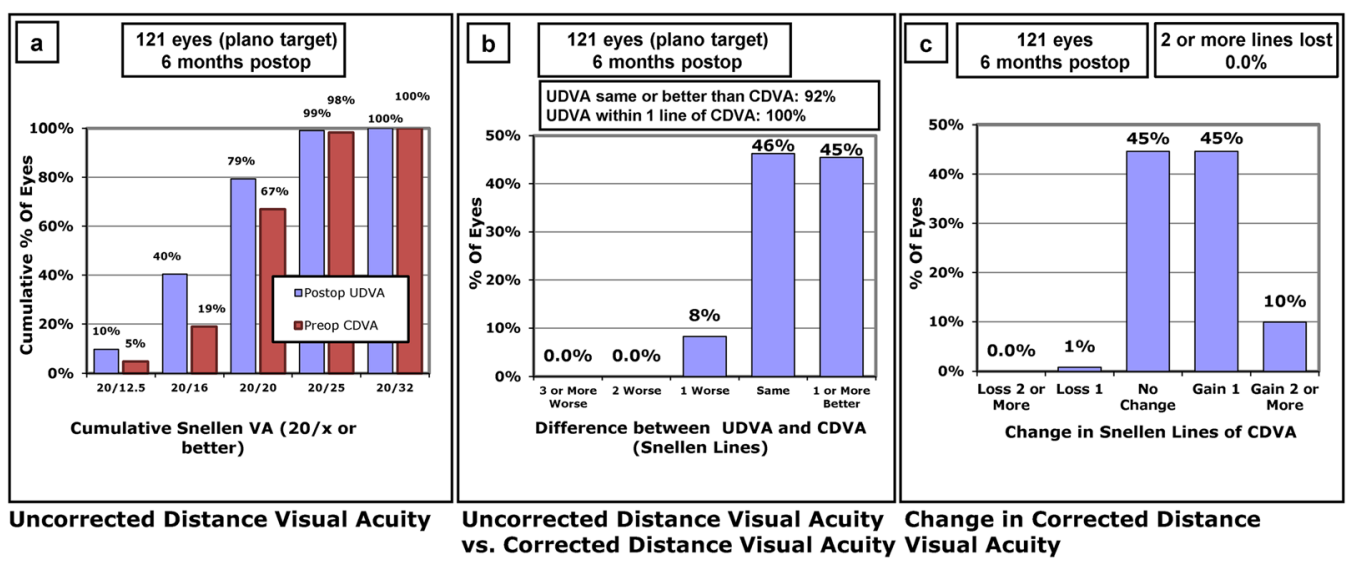

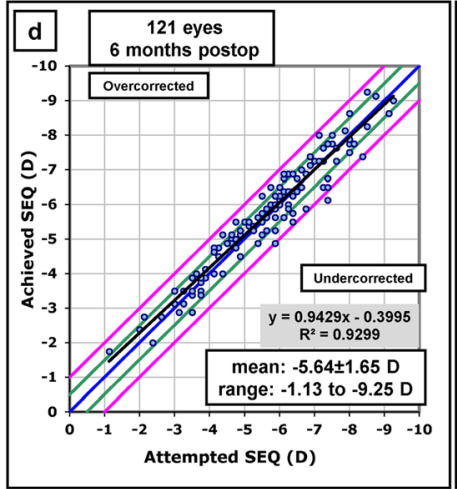

Spherical Equivalent Refraction Attempted vs Achieved

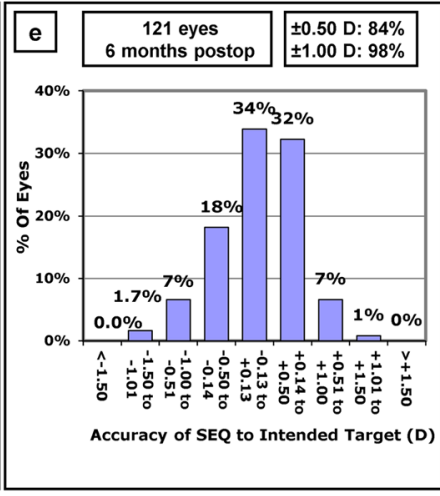

Spherical Equivalent Refraction Accuracy

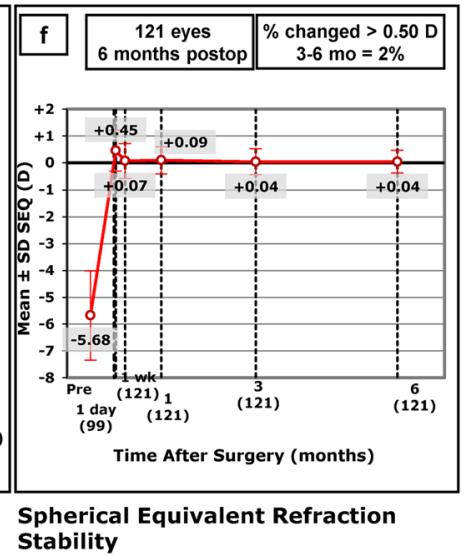

Stability

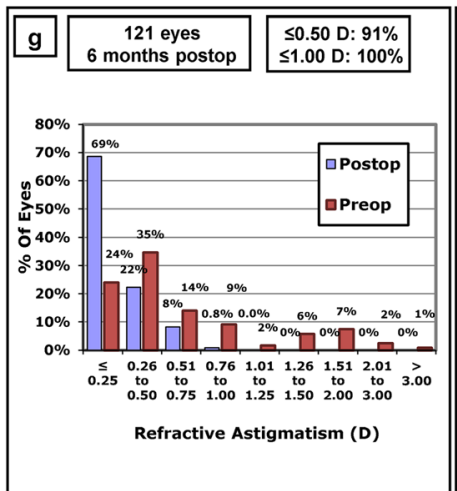

Refractive Astigmatism

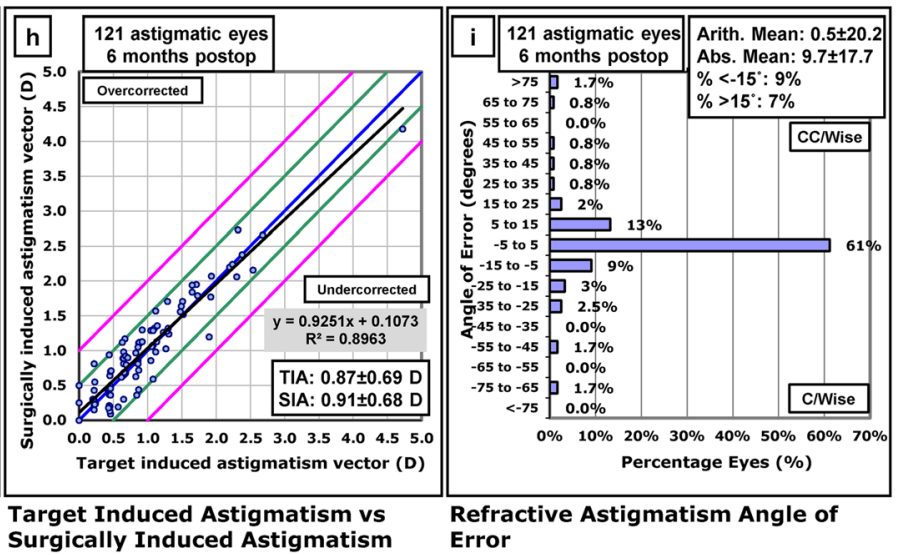

Fig. 1 Visual acuity and refraction 6 months post-surgery. a: UDVA, b: UDVA versus CDVA, c: Change in CDVA, d: SE Attempted versus Achieved, e: SE accuracy, $\mathbf{f}$ : SE stability, $\mathbf{g}$ : Refractive astigmatism, $\mathbf{h}$ : TIA versus SIA, i: Refractive astigmatism angle of error Abbreviations: UDVA = uncorrected distance visual acuity; CDVA = corrected distance visual acuity, $\mathrm{SE}=$ spherical equivalent; $\mathrm{SIA}=$ surgically induced astigmatism; TIA = target-induced astigmatism; Abs = absolute; Arith = arithmetic; $C$ Wise $=$ clockwise; CCMise $=$ counterclockwise 


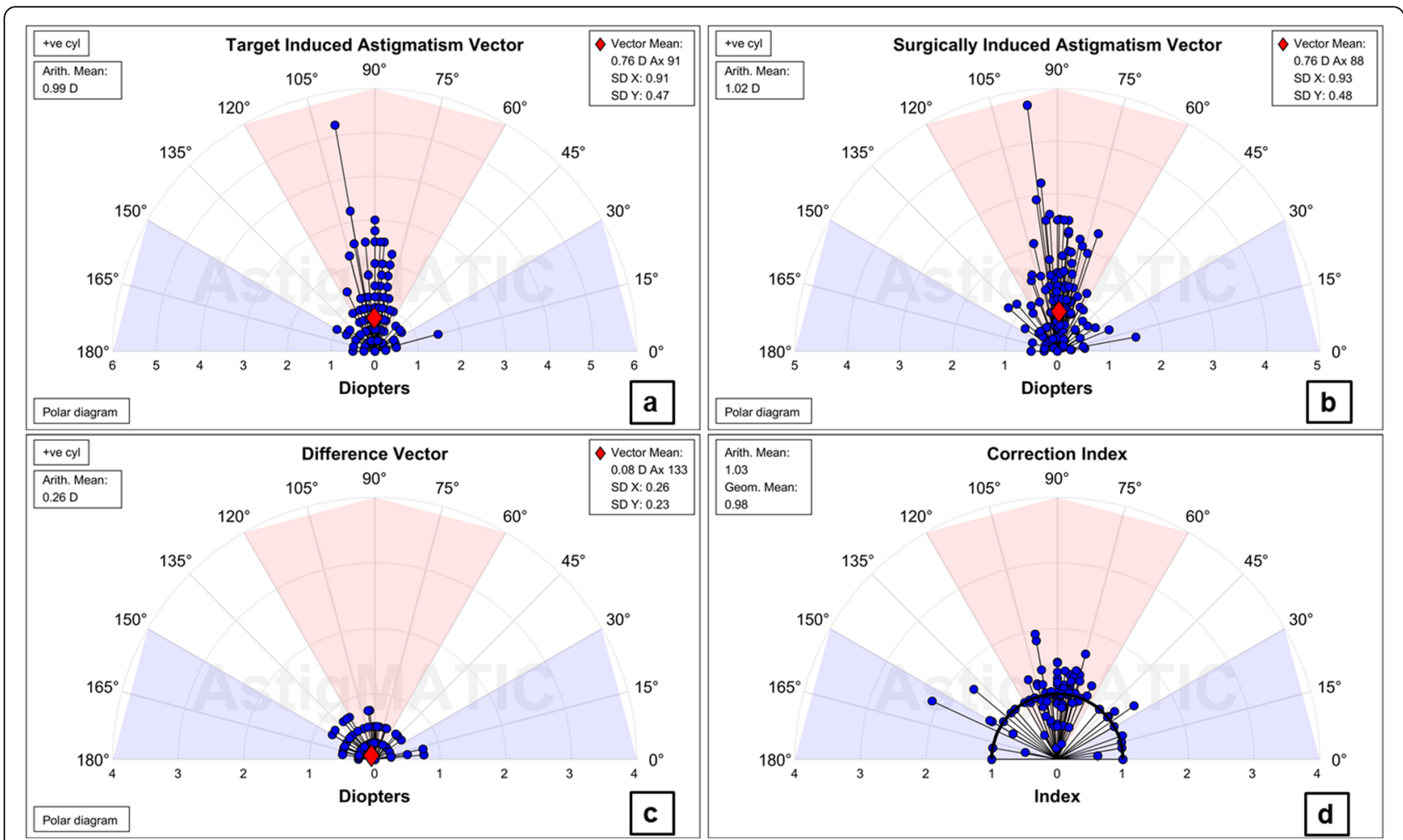

Fig. 2 Single-angle polar plots. a: Target induced astigmatism vector (TIA), b: Surgically induced astigmatism vector (SIA), c: Difference vector (DV), d: Correction index (Cl). The vector means are plotted as a red diamond (calculated in double-angle vector space) and the standard deviations for the $\mathrm{X}$ and $Y$ directions are displayed in the call-out box

achieved SE (Fig. 1d). The mean SE was $0.04 \pm 0.42 \mathrm{D}$ (range, $-1.25-1.13 \mathrm{D})$. Of the 121 eyes, 102 (84\%) were within $\pm 0.50 \mathrm{D}$ of the intended SE refraction, and 119 (98\%) were within $\pm 1.00 \mathrm{D}$ (Fig. 1e). Due to the bandage soft contact lens, refractive data were available for patients who underwent T-CAT LASEK 1-week postsurgery. The mean SE was stable from 3 to 6 months (Fig. 1f). The mean residual astigmatism was $-0.27 \pm$ $0.24 \mathrm{D}$ (range, $-1.00-0.00 \mathrm{D}$ ). The residual refractive astigmatism was $0.50 \mathrm{D}$ or less in 110 (91\%) eyes and $1.00 \mathrm{D}$ or less in 121 eyes (100\%) (Fig. 1g). There was an excellent correlation between surgically induced astigmatism (SIA) and target-induced astigmatism (TIA); the mean SIA was larger than the TIA (Fig. 1h). The mean absolute refractive astigmatism angle of error was $9.7 \pm$ 17.7 degrees, with 101 (83\%) eyes having an absolute angle of error of $\leq 15$ degrees (Fig. 1i). Single angle polar plots with vector means for TIA, SIA, difference vector (DV) and correction index (CI) at the 6-month followup are shown in Fig. 2.

\section{Optical quality and contrast sensitivity}

The optical quality was measured with OQAS. OSI, MTF, and SR were obtained preoperatively and at 6 months postoperatively (Table 4$)$. No significant difference $(p>$ 0.05) was found between the two measurements.

Photopic and scotopic contrast sensitivity at four spatial frequencies $(3-18 \mathrm{cpd})$ are shown in Fig. 3. Photopic contrast sensitivity did not change at these four spatial frequencies, while the scotopic contrast sensitivity at spatial frequencies of 3,6 and $12 \mathrm{cpd}$ significantly decreased 6 months postoperatively.

\section{Zernike analysis}

Since the transition zone affects aberrations, the Zernike analysis of the anterior corneal surface was performed with a Pentacam at an analysis diameter of $5.50 \mathrm{~mm}$, which was $0.5 \mathrm{~mm}$ lesser than the optical zone diameter.

Table 4 OQAS results obtained preoperatively and 6 months postop

\begin{tabular}{llll}
\hline & $\begin{array}{l}\text { Preoperatively } \\
(\text { Mean } \pm \text { SD) }\end{array}$ & $\begin{array}{l}\text { Postoperatively } \\
(\text { Mean } \pm \text { SD) }\end{array}$ & $p^{\text {a }}$ \\
\hline OSI & $0.50 \pm 0.45$ & $0.50 \pm 0.46$ & 0.662 \\
MTF & $33.51 \pm 9.72$ & $32.79 \pm 9.52$ & 0.466 \\
SR & $0.22 \pm 0.23$ & $0.22 \pm 0.19$ & 0.830 \\
\hline
\end{tabular}

a Paired t-test

$O Q A S=$ Optical Quality Analysis System II, OSI= objective scattering index, $M T F=$ modulation transfer function, $S R=$ Strehl ratio 

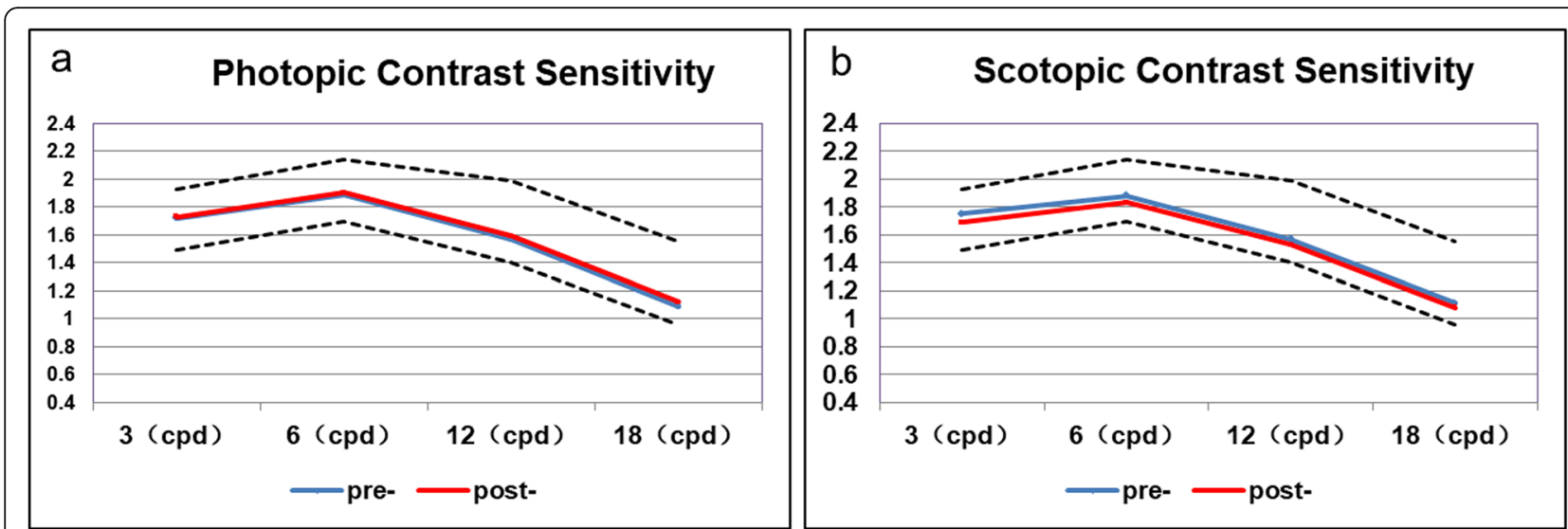

Fig. 3 Photopic (a) and scotopic (b) contrast sensitivities recorded preoperatively and 6 months postop

The root mean square (RMS) magnitudes of the total highorder aberration (HOA), astigmatism along $45^{\circ}(\mathrm{C} 3)$, astigmatism (C5), vertical coma (C7), horizontal coma (C8), and spherical aberration $(\mathrm{C} 12)$ were recorded. Variations in these parameters at different times are shown in Fig. 4. The total $\mathrm{HOA}, \mathrm{C} 3, \mathrm{C} 5$, and C7 significantly decreased $(p<0.05)$ and C12 significantly increased $(p<0.05) 1$ week post-surgery relative to the preoperative results (Fig. 3). However, total HOA, C7, and C8 significantly increased $(p<0.05) 6$ months postoperatively relative to the values obtained 1 week postoperatively. Follow-up examinations revealed that HOAs significantly increased without surgical intervention and that the variations mainly occurred between 1 week and 3 months postoperatively.

\section{Case example}

A 19-year-old man presented preoperatively with manifest refraction of the right eye of $-3.00 \mathrm{D}, 0 \mathrm{D} \times 0$

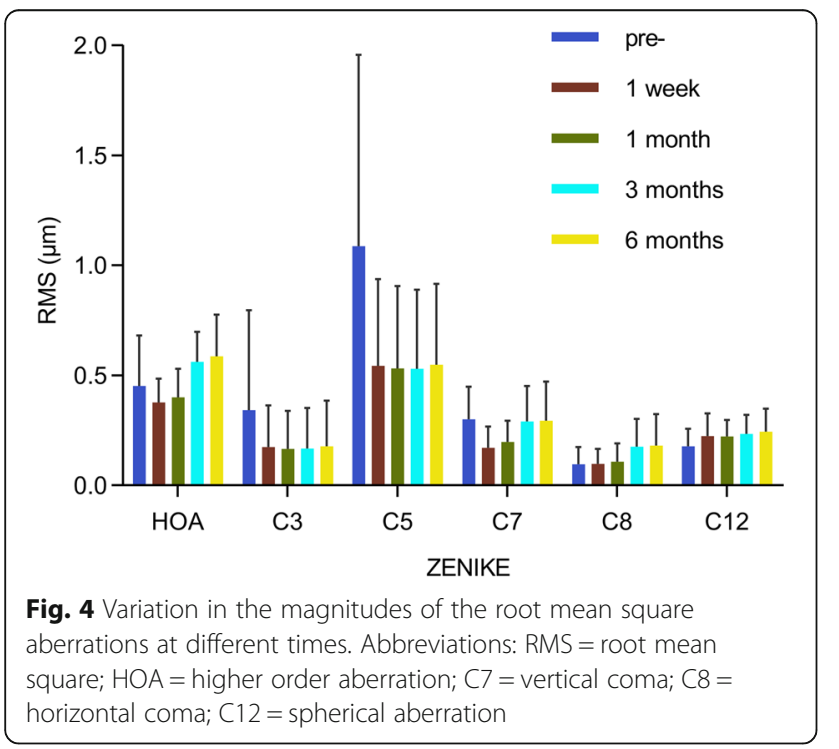

degree, and the corneal topographic astigmatism measured with the Topolyzer was $-1.03 \mathrm{D} \times 173$ degrees, while the UDVA was 20/63. We set the TMR data as the "target" (in this case, $-1.00 \mathrm{D} \times 173$ degrees), and the optometrist adjusted the manifest refractive power and axis to approach the "target" using the phoropter while paying attention to the change in SE. The CDVA under different refractive values is shown in Table 5 . When the astigmatism power exceeded $-0.50 \mathrm{D}$ or astigmatism axis exceeded 170 degrees, the CDVA significantly diminished, and the patient complained of discomfort. After adjusting the refraction back to $-3.00 \mathrm{D},-0.50$ $\mathrm{D} \times 170$ degrees, the CDVA did not decrease, and the patient experienced no discomfort. The correction data $(-3.00 \mathrm{D},-0.50 \mathrm{D} \times 170$ degrees) was then set using the topography-guided treatment planning software (Fig. 5). This case underwent T-CAT LASIK. Manifest refractive results and UDVA were $0.5 \mathrm{D},-0.25 \mathrm{D} \times 85$ degrees and

Table 5 Preoperative CDVA obtained under different refractive values in the case example

\begin{tabular}{ll}
\hline Refraction & CDVA (Snellen) \\
\hline$-3.00 \mathrm{D}$ & $20 / 40$ \\
$-2.50 \mathrm{D},-0.25 \mathrm{D} \times 170^{\circ}$ & $20 / 40$ \\
$-2.75 \mathrm{D},-0.25 \mathrm{D} \times 170^{\circ}$ & $20 / 32$ \\
$-3.00 \mathrm{D},-0.25 \mathrm{D} \times 170^{\circ}$ & $20 / 32$ \\
$-3.00 \mathrm{D},-0.50 \mathrm{D} \times 170^{\circ}$ & $20 / 20$ \\
$-2.75 \mathrm{D},-0.50 \mathrm{D} \times 170^{\circ}$ & $20 / 20$ \\
$-3.25 \mathrm{D},-0.50 \mathrm{D} \times 170^{\circ}$ & $20 / 32$ \\
$-3.00 \mathrm{D},-0.75 \mathrm{D} \times 170^{\circ}$ & $20 / 32$ \\
$-2.75 \mathrm{D},-0.75 \mathrm{D} \times 170^{\circ}$ & $20 / 32$ \\
$-3.00 \mathrm{D},-0.50 \mathrm{D} \times 20^{\circ}$ & $20 / 40$ \\
$-3.00 \mathrm{D},-0.50 \mathrm{D} \times 90^{\circ}$ & $20 / 63$ \\
$-3.00 \mathrm{D},-0.50 \mathrm{D} \times 140^{\circ}$ & $20 / 20$ \\
$-3.00 \mathrm{D},-0.50 \mathrm{D} \times 175^{\circ}$ & $20 / 32$ \\
\hline
\end{tabular}

CDVA $=$ corrected distance visual acuity 


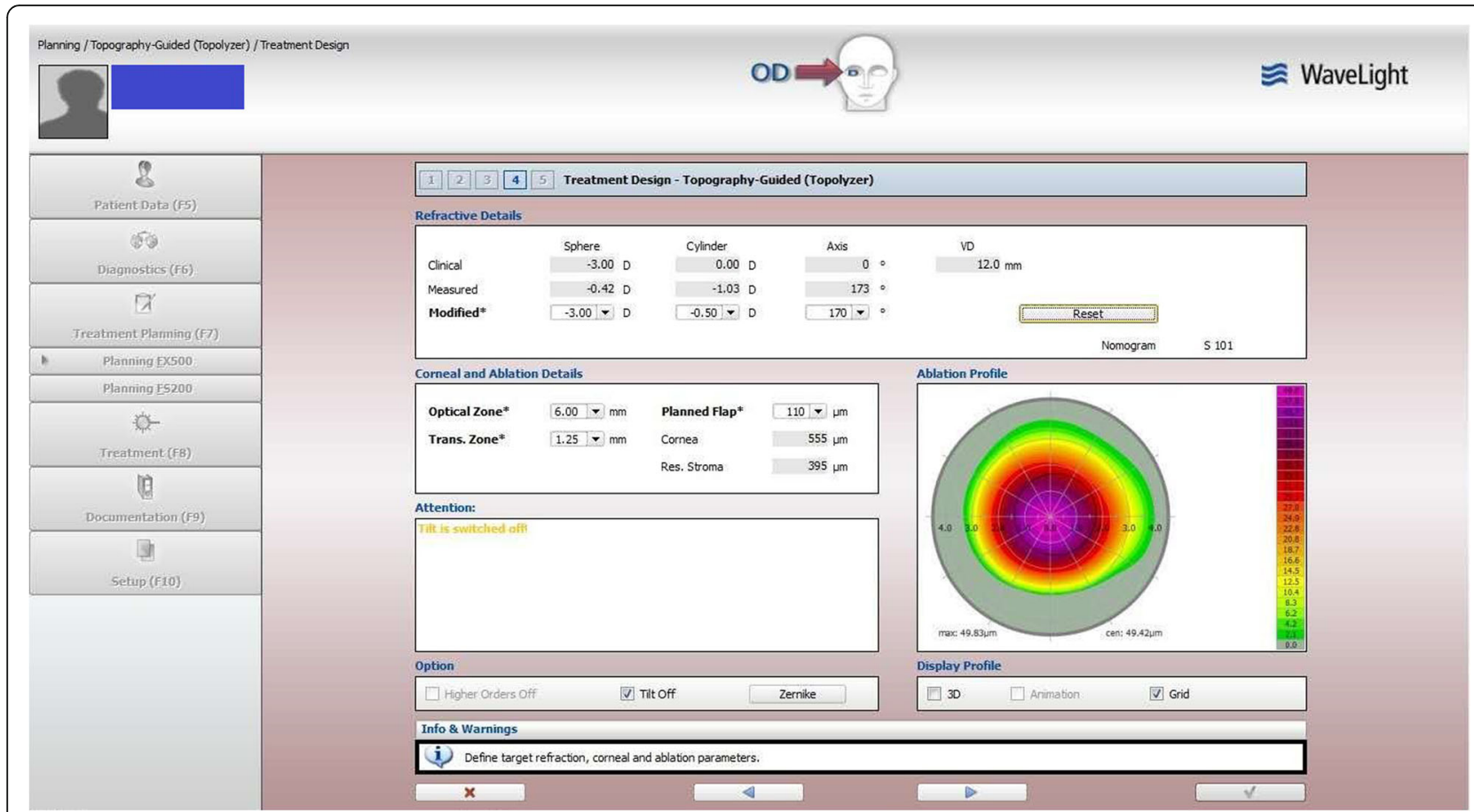

Fig. 5 Topography-guided treatment planning of the case example

20/12.5, respectively, 1 week post-surgery, $0.25 \mathrm{D},-0.25$ $\mathrm{D} \times 84$ degrees and $20 / 16$, respectively, 1 month postsurgery, $0.25 \mathrm{D},-0.25 \mathrm{D} \times 88$ degrees and 20/16, respectively, 3 months post-surgery, and $0.25 \mathrm{D},-0.25 \mathrm{D} \times 88$ degrees and 20/16, respectively, 6 months post-surgery. Corneal anterior surface curvature measured with a Pentacam preoperatively and 1 week postoperatively were compared (Fig. 6).

\section{Discussion}

MCA is a new method for correcting refractive errors calculated using data based on the manifest refraction and corneal topography and the patient's subjective perception.

Rather than referencing previous studies, the theory of refractive range was evaluated by clinical observation. We searched for theoretical support in previous studies on the visual system, which is composed of the optical and nervous systems; all optical systems exhibit blur to some extent [13], and blur perception is an elemental feature of the human visual system [14]. The field of perception reaches the psychological landscape, and perceptual decisions are based on a posterior probability distribution, which is obtained by combining the

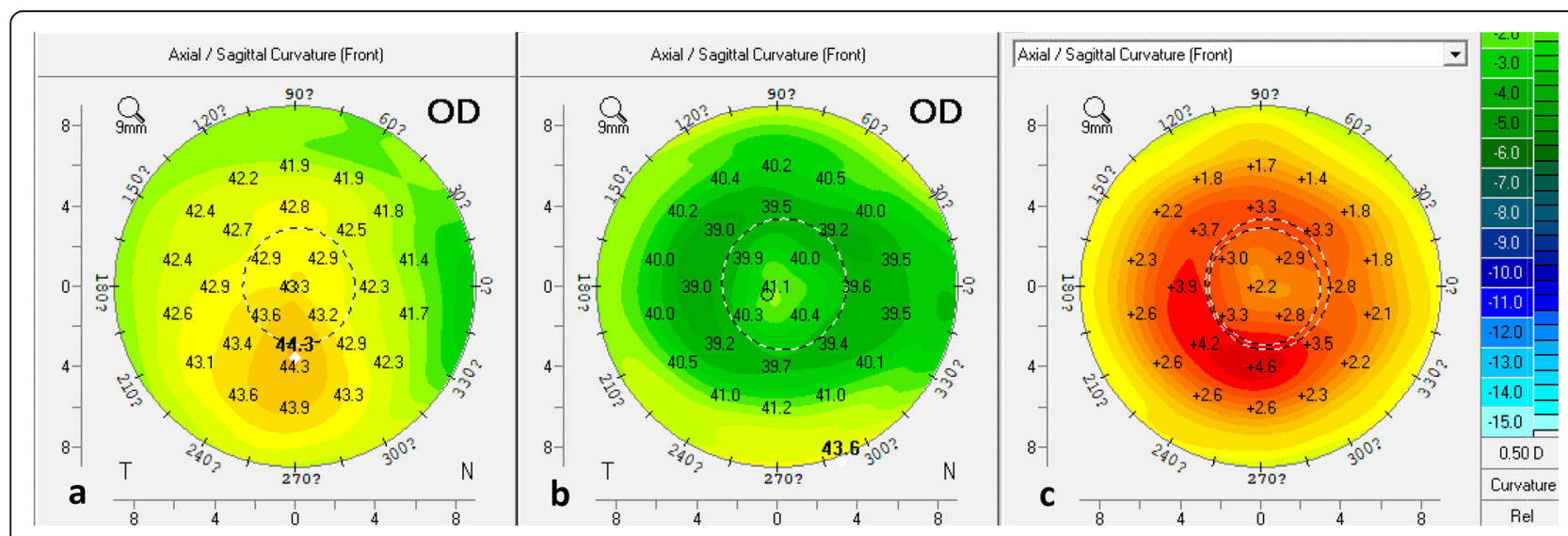

Fig. 6 Comparison of corneal anterior surface curvature recorded preoperatively and at 1 week postop for the case example. a: corneal anterior surface curvature preoperatively, b: corneal anterior surface curvature 1 week postoperatively, c: comparison between a and $\mathbf{b}$.? means degree 
likelihood and prior distributions [15]. This combination produced the most mathematically reliable estimate: the maximum likelihood estimate (MLE). The MLE can be obtained from blurred retinal images within a refractive range and the CDVA could thus be achieved within a refractive range rather than as a fixed refractive value. Within the refractive range, there was no significant difference in a patient's subjective perception.

The CV includes importing treatment data into the laser treatment planning model for T-CAT. The application of topography-guided treatments with this platform have been extensively reported $[7,8]$.

The FDA algorithm is the most commonly used planning algorithm applied to CV. TMR or LYRA protocols use the astigmatic data measured via corneal topography as the correction for astigmatism. Multiple factors cause manifest refraction and topographic astigmatism to differ: anterior corneal higher-order aberrations, posterior corneal astigmatism, anterior and posterior lenticular astigmatisms, refractive index variability, decentration of the crystalline lens, retinal tilt, and cortical perception [16]. When the posterior corneal surface and intraocular astigmatism are large, astigmatism correction based on corneal topography alone may cause an increase in total eye aberration or corneal astigmatism and tilting of intraocular astigmatism. A recent study [9] also suggested that the TMR and LYRA protocols have drawbacks for correcting ametropia.

Many studies have shown excellent outcomes when planning treatment based solely on subjective manifest refractive astigmatism $[9,17-23]$. However, only one study [9] reported differences between manifest astigmatism and topographic astigmatism. The inclusion criteria for most studies did not consider the differences in preoperative power or axis (or both) of the astigmatism between the manifest refraction and the corneal topographic data.

Despite the natural optimal comfort with manifest refraction, this refraction was in a range rather than being a fixed value. We tried to find the correction value closest to the topographic astigmatism within the refractive range with which the patient was comfortable. Combined with the patient's subjective feelings, MCA maximally corrects the irregularities of the anterior surface of the cornea to achieve a balance between correction of the irregular cornea and the patient's subjective feelings.

LASEK is applied for correction of low to moderate myopia and thin corneas, and there is uncertainty in how LASEK compares with LASIK in achieving better refractive and visual results [24]. Hence, we put together the results of LASEK and LASIK.

Postoperative results of visual acuity and refraction demonstrated that MCA is safe, effective, accurate, predictable, and stable. The vector analysis of astigmatism (Figs. 1 and 2) showed good results for astigmatic correction. In the present study, one line of vision was gained in $40 \%$ of eyes, and two lines were gained in $10 \%$. Additionally, the residual refractive astigmatism $(0.50 \mathrm{D}$ or less in $91 \%$ eyes and $1.00 \mathrm{D}$ or less in $100 \%$ ) was consistent with that in two studies in which treatment was based directly on subjective manifest refraction [20, 23]. These findings demonstrate the feasibility of MCA.

The present study found that at 6 months postop, the OQAS results showed no improvement in optical quality, no change in photopic contrast sensitivity, and significant decreases in scotopic contrast sensitivity at spatial frequencies of 3,6 , and $12 \mathrm{cpd}$. Postoperative corneal HOAs may partly account for the lack of improvement in optical and visual quality.

Repeatability and reproducibility of Zernike measurements on the Pentacam have been reported [25]. Thirdorder aberrations rather than total aberrations are the main factors affecting contrast sensitivity, and $\mathrm{C} 7$ is reportedly negatively correlated with contrast sensitivity and visual quality [26]. In the present study, total HOA and C7 significantly decreased 1 week post-surgery, indicating that the procedures reduced irregularities in the corneas. However, the $\mathrm{HOA}, \mathrm{C} 7$, and $\mathrm{C} 8$ significantly increased during follow-up. Due to the increase in pupil diameter in darkness, scotopic contrast sensitivity may be more susceptible to the increase in corneal wavefront aberration, and thus, account for the postoperative decrease in scotopic contrast sensitivity.

Notably, HOAs increased during follow-up without surgical intervention. This change may be associated with non-uniform proliferation of the corneal epithelium. Two recent studies reported remodeling of the corneal epithelium following corneal refractive surgery $[27,28]$. The LYRA protocol also mentions the effect of the epithelium on HOA. Non-uniform proliferation of the corneal epithelium increases the irregularities on the corneal surface and alters the aberrations themselves. Other factors, such as tear film, corneal biomechanics, and intraocular pressure, may also affect the HOA. Optical and visual quality data obtained 1 week postop would have yielded more insights. Nevertheless, further study on the variation in HOAs is needed.

Finally, our study indicated that the selection of the planning algorithm for the $\mathrm{CV}$ system could be conducted in the following order: (1) FDA algorithm, (2) MCA can be used as an alternative to current protocols when a patient exceeds the application conditions of the FDA algorithm and the posterior corneal surface or intraocular astigmatism is large.

\section{Conclusion}

MCA was shown to be safe, efficacious, accurate, predictable, and stable. It is a feasible method and can be used as a complementary to T-CAT. 


\section{Abbreviations}

T-CAT: Topography-guided custom ablation treatment; MCA: Mutual comparative analysis; FDA: The U.S. Food and Drug Administration; CV: Contoura vision; LASIK: Laser-assisted in situ keratomileusis; TMR: Topography-modified refraction; LYRA: Layer Yolked Reduction of Astigmatism; LASEK: Laser-assisted subepithelial keratectomy; ORA: Oculus residual astigmatism; OSI: Objective scattering index; MTF: Cutoff for modulation transfer function; SR: Strehl ratio; OQAS: Optical quality analysis system; BCDVA: Best-corrected distance visual acuity; SE: Spherical equivalent; UDVA: Uncorrected distance visual acuity; SIA: Surgically induced astigmatism; TIA: Target-induced astigmatism; DV: Difference vector; Cl: Correction index; RMS: Root mean square; C7: Vertical coma; C8: Horizontal coma; C12: Spherical aberration; HOA: High order aberration; MLE: Maximum likelihood estimate

\section{Acknowledgements}

Not applicable.

\section{Authors' contributions}

TLiu and JB contributed to conception, design, and interpretation of data. KWC contributed to data acquisition and drafted the article. LL and TZ contributed to data acquisition. All authors approved the final version of the manuscript.

\section{Funding}

This study was not funded.

\section{Availability of data and materials}

The datasets used and/or analyzed during the current study are available from the corresponding author on reasonable request.

\section{Ethics approval and consent to participate}

This study was approved by the Ethics Committee of Daping Hospital and the Research Institute of Surgery, the Army Medical University of PLA (Chong Qing, China). The experiment complied with the Declaration of Helsinki and written informed consent was obtained from all patients. The clinical registration number is ChiCTR2000030193.

\section{Competing interests}

The authors declare that they have no competing interests.

\section{Author details}

'77120 unit of People's Liberation Army (PLA), No.1441 FengJu Ring Road, Jinniu District, Chengdu 610081, China. ${ }^{2}$ Chongqing vision institute, No. 77 Changjiang 2 Road, Yuzhong District, Chongqing 400042, China. ${ }^{3}$ Department of Ophthalmology, Daping Hospital and the Research Institute of Surgery of the Army Medical University of PLA, No. 10 Changjiangzhi Road, Yuzhong District, Chongqing 400042, China.

Received: 3 October 2019 Accepted: 15 June 2020

Published online: 07 July 2020

\section{References}

1. Merchea M. Customized corneal ablation: the quest for supervision. Optom Vis Sci. 2001;78(11):780-1.

2. Holland S, Lin DT, Tan JC. Topography-guided laser refractive surgery. Curr Opin Ophthalmol. 2013;24(4):302-9.

3. Kanellopoulos AJ. Topography-guided custom retreatments in 27 symptomatic eyes. J Refract Surg. 2005:21(5):S513-8.

4. Yoshida Y, Nakamura T, Hara S, Yoshida N, Kojima T, Ichikawa K. Topography-guided custom ablation for irregular corneal astigmatism using the NIDEK NAVEX laser system. J Refract Surg. 2008;24(1):24-32.

5. Shetty R, D'Souza S, Srivastava S, Ashwini R. Topography-guided custom ablation treatment for treatment of keratoconus. Indian J Ophthalmol. 2013; 61(8):445-50.

6. Summary of Safety and Effectiveness Data [SSED]. Available from: http:// www.accessdata.fda.gov/cdrh_docs/pdf2/P0200505012b.pdf.

7. Kanellopoulos AJ. Topography-modified refraction (TMR): adjustment of treated cylinder amount and axis to the topography versus standard clinical refraction in myopic topography-guided LASIK. Clin Ophthalmol. 2016;10: 2213-21.
8. Motwani M. The use of WaveLight ${ }^{\circledR}$ Contoura to create a uniform cornea: the LYRA protocol. Part 3: the results of 50 treated eyes. Clin Ophthalmol. 2017;11:915-21.

9. Wallerstein A, Gauvin M, Qi SR, Bashour M, Cohen M. Primary topographyguided LASIK: treating manifest refractive astigmatism versus topographymeasured anterior corneal astigmatism. J Refract Surg. 2019;35(1):15-23.

10. Alpins NA. New method of targeting vectors to treat astigmatism. J Cataract Refract Surg. 1997;23(1):65-75.

11. Reinstein DZ, Archer TJ, Randleman JB. JRS standard for reporting astigmatism outcomes of refractive surgery. J Refract Surg. 2014;30(10):654-9.

12. Gauvin M, Wallerstein A. AstigMATIC: an automatic tool for standard astigmatism vector analysis. BMC Ophthalmol. 2018;18(1):255

13. Watson $A B$, Ahumada AJ. Blur clarified: a review and synthesis of blur discrimination. J Vis. 2011;11(5):10

14. Maiello G, Walker L, Bex PJ, Vera-Diaz FA. Blur perception throughout the visual field in myopia and emmetropia. J Vis. 2017;17(5):3

15. Beierholm UR, Quartz SR, Shams L. Bayesian priors are encoded independently from likelihoods in human multisensory perception. J Vis. 2009;9(5):23.1-9.

16. Wallerstein A, Gauvin M, Cohen M. WaveLight ${ }^{\circledR}$ Contoura topographyguided planning: contribution of anterior corneal higher-order aberrations and posterior corneal astigmatism to manifest refractive astigmatism. Clin Ophthalmol. 2018;12:1423-6.

17. Hashmani S, Hashmani $\mathrm{N}$, Haroon $\mathrm{H}$, Hashmi Y. Visual and refractive outcomes of topography-guided laser-assisted in situ keratomileusis in virgin eyes. Cureus. 2018;10(1):e2131.

18. Kim J, Choi SH, Lim DH, Yang CM, Yoon GJ, Chung TY. Topography-guided versus wavefront-optimized laser in situ keratomileusis for myopia: surgical outcomes. J Cataract Refract Surg. 2019;45(7):959-65.

19. Zhang $Y$, Chen $Y$. A randomized comparative study of topography-guided versus wavefront-optimized FS-LASIK for correcting myopia and myopic astigmatism. J Refract Surg. 2019;35(9):575-82.

20. Faria-Correia F, Ribeiro S, Monteiro T, Lopes BT, Salomão MQ, Ambrosio R Jr. Topography-guided custom photorefractive keratectomy for myopia in primary eyes with the WaveLight EX500 platform. J Refract Surg. 2018;34(8): 541-6.

21. Tiwari NN, Sachdev GS, Ramamurthy S, Dandapani R. Comparative analysis of visual outcomes and ocular aberrations following wavefront optimized and topography-guided customized femtosecond laser in situ keratomileusis for myopia and myopic astigmatism: a contralateral eye study. Indian J Ophthalmol. 2018;66(11):1558-61.

22. Shetty R, Shroff R, Deshpande K, Gowda R, Lahane S, Jayadev C. A prospective study to compare visual outcomes between wavefrontoptimized and topography-guided ablation profiles in contralateral eyes with myopia. J Refract Surg. 2017;33(1):6-10

23. Stulting RD, Fant BS, Group TCS, Bond W, Chotiner B, Durrie D, et al. Results of topography-guided laser in situ keratomileusis custom ablation treatment with a refractive excimer laser. J Cataract Refract Surg. 2016:42(1):11-8.

24. Kuryan J, Cheema A, Chuck RS. Laser-assisted subepithelial keratectomy (LASEK) versus laser-assisted in-situ keratomileusis (LASIK) for correcting myopia. Cochrane Database Syst Rev. 2017;2:CD011080.

25. McAlinden C, Schwiegerling J, Khadka J, Pesudovs K. Corneal aberrations measured with the oculus Pentacam HR: repeatability and reproducibility. J cataract refract Surg. 2020;46(4):581-90.

26. Zhao PF, Li SM, Lu J, Song HM, Zhang J, Zhou YH, et al. Effects of higherorder aberrations on contrast sensitivity in normal eyes of a large myopic population. Int J Ophthalmol. 2017;10(9):1407-11.

27. Fan L, Xiong L, Zhang B, Wang Z. Longitudinal and regional non-uniform remodeling of corneal epithelium after topography-guided FS-LASIK. J Refract Surg. 2019;35(2):88-95.

28. Kanellopoulos AJ. Comparison of corneal epithelial remodeling over 2 years in LASIK versus SMILE: a contralateral eye study. Cornea. 2019;38(3):290-6. 